\section{Cancer disparities}

There is increasing recognition that diverse populations should be included in cancer genomics and epigenomics studies to identify differences within these populations that might account for disparities in cancer incidence and survival. To date, much of our knowledge of the genetic features of cancer comes from analysis of samples from The Cancer Genome Atlas (TCGA), which mostly comprises tumours derived from patients with European ancestry. To address this shortcoming, Sinha et al. set about systematically mapping genomic alterations in lung tumours from an ethnically unbiased patient cohort.

Using 222 lung tumour samples from the National Cancer InstituteMaryland (NCI-MD) study, the researchers produced genome-wide copy-number profiles. This revealed that lung squamous cell carcinomas (LUSCs) but not lung adenocarcinomas (LUADs) from African Americans (AAs) had higher genomic instability (GI) than European extent in LUADs.
Americans (EAs). Likely accounting for this difference in GI in LUSCs was a higher degree of homologous recombination deficiency (HRD). Furthermore, complex structural variants associated with HRD, such as chromothripsis, were more common in LUSCs from AAs than EAs and to a lesser

Probing the differences in somatic copy-number alterations (SCNAs) in more detail revealed that lung cancer subtype-specific SCNAs at the level of the chromosome arm were detected at a higher frequency in tumours from AAs than EAs. Furthermore, eight novel AA-specific focal-level - defined as shorter than half a chromosome arm - SCNAs were identified. Recurrent features at the gene level included KRAS amplification and PTEN deletion occurring more often in LUSCs from AAs than EAs.

Importantly, observations made within the NCI-MD cohort were validated with analysis of TCGA, despite the relatively small number of samples from AAs in this cohort.

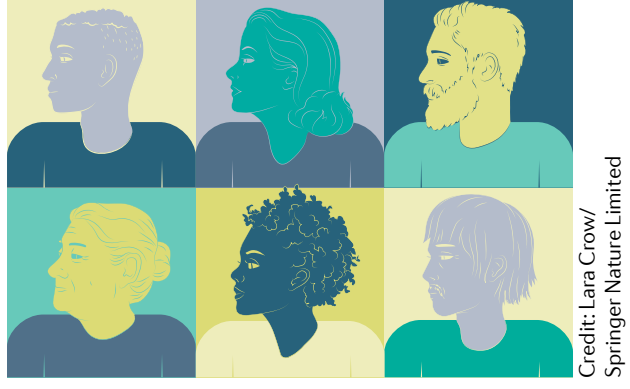

Extending their analysis to more cancer types by mining TCGA SCNA profiles of 6,492 pan-cancer samples showed a general trend for higher levels of GI, HRD and chromothripsis across multiple tumour types from AAs. The increased germline HRD noted in the pan-cancer samples and LUSCs from AAs might underlie these somatic differences.

This work calls for more studies powered to compare differences by ancestry groups to help reduce worldwide cancer burden.

Anna Dart

ORIGINAL ARTICLE Sinha, S. et al. Higher prevalence of homologous recombination deficiency in tumors from African Americans versus European Americans. Nat. Cancer 1, 112-121 (2020)

RELATED ARTICLE Teh, B. T. et al. The importance of including diverse populations in cancer genomic and epigenomic studies. Nat.Rev.Cancer 19, 361-362 (2019)

\section{CANCER IMMUNOTHERAPY}

\section{Starving tumours}

Fasting-like conditions can improve the efficacy of chemotherapy by eliciting an antitumour immune response. Therefore, it might be reasoned that the combination of fasting and immunotherapy would serve to increase tumour immunogenicity and counteract primary resistance to immune checkpoint blockade (ICB). Ajona et al. explored this possibility.

To test the effect of the combination treatment, syngeneic mouse models of lung cancer were used wherein two different tumour cell lines 393P and LLC - were subcutaneously implanted into mice. Once tumours were established, fasting in the form of short-term starvation (STS) was carried out by withdrawing access to food during set intermittent cycles, along with administration of a PD1 antibody. STS synergized with PD1 blockade to attenuate tumour growth and increase the survival of the mice. $\mathrm{CD} 8^{+} \mathrm{T}$ cells are likely to mediate the sensitization of lung tumour cells to the action of PD1 blockade under conditions of STS as depletion of this effector cell type abolished the antitumour effect of the combination. In the case of the 393P tumour model, mice that exhibited complete responses were protected from tumour re-challenge, consistent with induction of immunological memory. Furthermore, substitution of the PD1 antibody for a PDL1 antibody in the combination treatment was also able to inhibit LLC tumour growth. Metabolic changes associated with fasting include lower systemic levels of insulin-like growth factor 1 (IGF1). In agreement with this, STS either with or without anti-PD1 reduced plasma IGF1 levels in tumour-bearing mice. Interestingly, lower levels of circulating IGF1 or low tumour expression of the IGF1 receptor (IGF1R) correlated with durable clinical benefit in patients with non-small-cell lung cancer treated with either anti-PD1 or anti-PDL1 therapy.

In turn, this led the authors to assess the effect of anti-PD1 in conjunction with an IGF1R antagonist. The reduced tumour growth observed with this co-treatment correlated with increased numbers of tumour-infiltrating $\mathrm{CD}^{+}{ }^{+}$and $\mathrm{CD} 4^{+}$ $T$ cells with less expression of exhaustion markers and decreased numbers of intratumoural $\mathrm{T}_{\text {reg }}$ cells.

Overall, this study supports the clinical testing of IGF1R inhibitors in combination with ICB.

Anna Dart

ORIGINAL ARTICLE Ajona, D. et al. Short-term starvation reduces IGF-1 levels to sensitize lung tumors to PD-1 immune checkpoint blockade. Nat. Cancer 1, 75-85 (2020) RELATED ARTICLE Nencioni, A. et al. Fasting and cancer: molecular mechanisms and clinical application. Nat.Rev. Cancer 18, 707-719 (2018) 